\title{
Le Butard et la Muette : deux pavillons de chasse d'Ange-Jacques Gabriel pour Louis XV
}

Le Butard and La Muette: two hunting lodges by Ange-Jacques Gabriel for Louis XV

Marie-Marguerite Roy

\section{(2) OpenEdition}

Journals

Édition électronique

URL : http://journals.openedition.org/cel/313

DOI : $10.4000 / \mathrm{cel} .313$

ISSN : 2262-208X

Éditeur

École du Louvre

Référence électronique

Marie-Marguerite Roy, "Le Butard et la Muette : deux pavillons de chasse d'Ange-Jacques Gabriel pour Louis XV », Les Cahiers de l'École du Louvre [En ligne], 6 | 2015, mis en ligne le 01 avril 2015, consulté le 17 septembre 2019. URL : http://journals.openedition.org/cel/313; DOI : 10.4000/cel.313

\section{(c)}

Les Cahiers de l'École du Louvre sont mis à disposition selon les termes de la licence Creative Commons Attribution - Pas d'Utilisation Commerciale - Pas de Modification 4.0 International. 


\section{Le Butard et la Muette : \\ deux pavillons de chasse \\ d'Ange-Jacques Gabriel pour Louis XV}

\section{Marie-Marguerite Roy}

Parmi les nombreux rendez-vous de chasse construits sous les règnes de Louis XV et de Louis XVI dans l'ouest parisien, et dont très peu subsistent aujourd'hui, plusieurs sont l'œuvre ou sont attribués au Premier Architecte du Roi Ange-Jacques Gabriel. Toujours existants, les pavillons du Butard et de la Muette ont été édifiés dans les bois de La Celle Saint-Cloud et en forêt de SaintGermain-en-Laye. Malgré cette distance géographique, il existe des liens étroits dans le déroulement de leur construction respective et dans leur utilisation à travers les régimes politiques successifs. Retracer leur histoire est un travail en grande partie inédit ${ }^{1}$, conséquence de l'absence d'études approfondies et récentes. Seul le Butard a fait l'objet de deux articles dans les années $1930^{2}$, tandis que l'histoire de la Muette était jusque-là restée dans les archives qui constituent donc la source principale, sinon unique, d'informations ${ }^{3}$.

Quels sont les liens qui unissent ces deux pavillons de chasse, tant du point de vue de leur architecture, de leur topographie, de leur décor, que de leur ameublement, leur évolution a-t-elle été linéaire et simultanée ? Émanations du pouvoir royal, mais ayant échappé aux destructions de la Révolution, quel regard les régimes du $\mathrm{XIX}^{\mathrm{e}}$ siècle ont-ils porté sur eux ?

Premier construit, le pavillon du Butard adopte une architecture originale et novatrice, qui le place à mi-chemin entre le repos de chasse et l'ermitage. Satisfait de cette disposition avantageuse, Louis XV commande à Ange-Jacques Gabriel un projet similaire: le pavillon de la Muette. La passion pour la chasse des dirigeants $\mathrm{du} \mathrm{XIX}^{\mathrm{e}}$ siècle les réaffecte dans leur fonction première après la période révolutionnaire.

\section{Le pavillon du Butard, repos de chasse ou lieu de retraite?}

Premier de la longue liste des rendez-vous de chasse de l'ouest parisien, le pavillon du Butard est édifié à partir de juillet 1750. Disposé sur une butte légèrement remblayée ${ }^{4}$ au milieu d'un massif boisé, son accès se fait par une allée rectiligne terminée par une esplanade qui facilite la circulation des voitures. La vue pratiquement panoramique depuis le pavillon résulte de l'association de la construction en surplomb et du plan en "T » de l'édifice. Bâti sur caves, il se présente sous la forme d'un bâtiment rectangulaire à avant-corps en légère saillie abritant le vestibule, le réchauffoir (ou cuisine particulière du Roi) à droite, et la garde-robe doublée de l'escalier vers l'attique à gauche; cet attique abrite trois petites pièces, dont deux chambres. Une forte saillie octogonale sur la façade arrière abrite le salon à l'italienne, circulaire à l'intérieur, et ouvrant par trois grandes portes-fenêtres cintrées et deux baies rectangulaires. À l'aplomb de la butte, vaste et largement ouvert sur l'extérieur, il s'agit de la pièce principale, où se rassemblent le roi et ses invités (fig. 1 et 2). Ange-Jacques Gabriel signe avec le

1. Ce travail a fait l'objet du mémoire de recherche de Master 2 de l'École du Louvre en 2012 , sous le titre Les pavillons de chasse du Butard et de la Muette, de leur construction à la fin de la Monarchie de Juillet, présenté sous la direction de Jean-Jacques Gautier, inspecteur au Mobilier national, et avec la participation d'Élisabeth Caude, conservateur au Château de Versailles.

2. Maurice Noury, «Recherches sur Le Butard », Bulletin de la Société archéologique Historique et Artistique de Marly-le-Roi, 1938, t. I, n 7, pp. 196-198 ; 1939, t. I, nº 8, pp. 237-240 ; 1943, t. I, pp. 295-297.

3. Pour les généralités, on consultera la monographie de Michel Gallet et Yves Bottineau, Les Gabriel, Paris, Picard, 2004 (les auteurs y dressent une liste des pavillons, aujourd'hui à réviser), et celle de Christopher Tadgell, Ange-Jacques Gabriel, Londres, A. Zwemmer Ltd, 1978. Sur la question de l'architecture, on consultera Louis Hautecœur, Histoire de l'architecture classique en France, t. III, Paris, A. et J. Picard et Cie, 1950.

4. Paris, Archives nationales $\mathrm{O}^{1} 1870(5)-21$ et -4. 
pavillon du Butard un des premiers édifices affichant son style si caractéristique et menant au néoclassicisme du règne de Louis XVI : les façades sont entièrement recouvertes de bossages continus, les baies en plein cintre sont inscrites dans un renfoncement rectangulaire et surmontées d'une agrafe à clef. À travers ces quelques éléments, le Butard se place dans la suite immédiate du pavillon Français de Trianon (1749), qui résume le style de Gabriel. Une table de pierre est disposée au-dessus de chaque petite porte latérale ; elle semble avoir supporté à l'origine un trophée de tête de cerf en ronde-bosse qui devait rappeler ceux de la Cour des Cerfs du château de Versailles; la date de leur disparition n'est pas connue ${ }^{5}$. Le fronton de la façade d'entrée est sculpté d'une scène de chasse. Si l'on met à part le rendez-vous de chasse de Saint-Hubert (1755), qui de pavillon est devenu un véritable château, le Butard fait figure d'exception parmi les autres rendez-vous : en effet, lui seul possède une décoration extérieure poussée et thématique qui précise la vocation cynégétique du bâtiment.

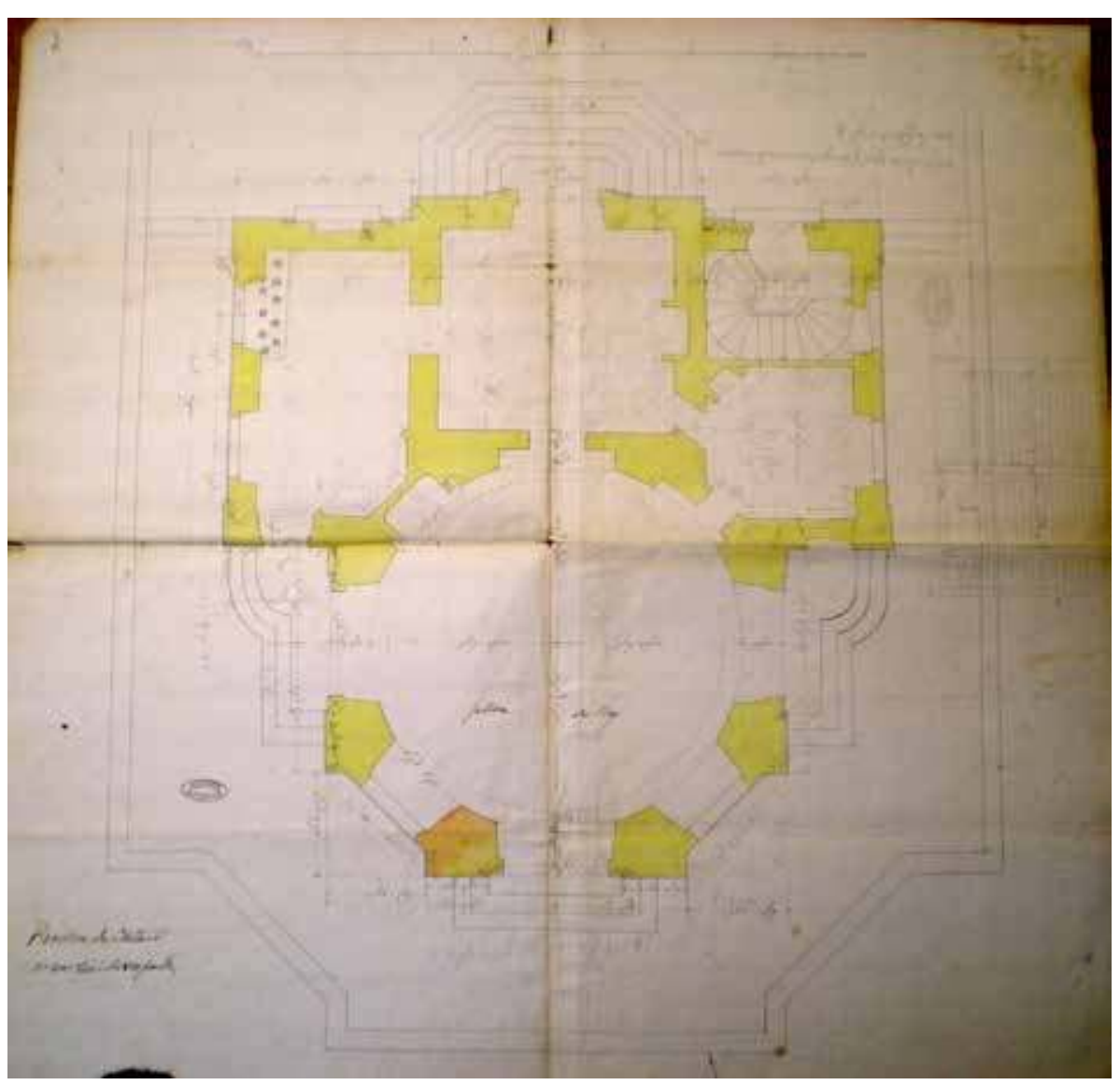

Ce décor cynégétique extérieur contraste avec la décoration intérieure, totalement achevée en 1752. Des boiseries originales, brûlées en 1870, ne restent que les corniches des pièces du rez-de-chaussée. Elles avaient été réalisées par le menuisier Bourgeois, sculptées par Jules-Antoine Rousseau, et peintes par Louis Pollevert. Ce même thème cynégétique est décliné dans les scènes sculptées, mais elles revêtent cependant à l'intérieur du pavillon un ton plus charmant, grâce à l'utilisation de putti et d'animaux évoluant dans des rinceaux. Les précieux encadrements chantournés des glaces ont été exécutés par Rousseau, associé ici au doreur Gobert, et le travail de serrurerie est l'œuvre de Louis Gamain. On peut constater que les entrepreneurs sollicités sont d'importants artistes travaillant au château de Versailles : on doit notamment à Jules-Antoine Rousseau les sculptures du cabinet du Conseil et à Louis Gamain les ferronneries de l'escalier du Petit Trianon. 
Ainsi bâti, le pavillon du Butard évoque autant le rendez-vous de chasse que le pavillon d'agrément, dont l'ermitage est une émanation. Dans ses mémoires, le marquis d'Argenson désigne par ailleurs le Butard et d'autres rendez-vous par le nom d'" ermitage ». Le Dictionnaire de l'Académie (1762) définit l'ermitage comme : " une maison écartée et champêtre ». Ce rapprochement évident entre
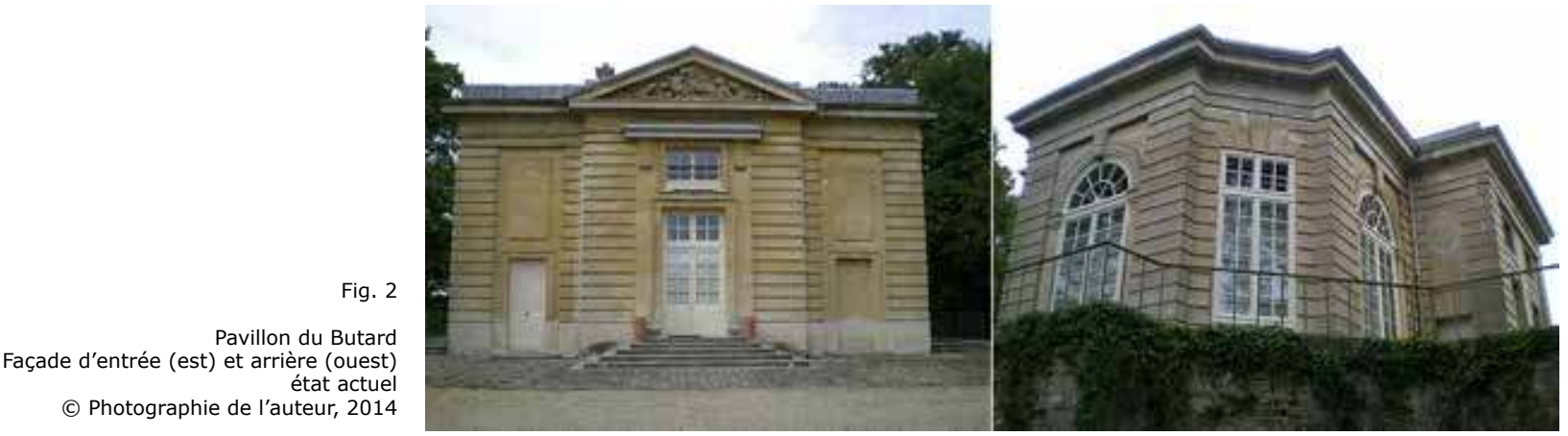

les deux fonctions est soutenu par plusieurs facteurs. D'abord, par sa situation géographique : il est à l'écart, sans cependant être trop éloigné de Versailles (à une lieue), mais d'accès commode, et s'ouvre largement sur l'extérieur ; il dispose aussi de vastes communs ${ }^{6}$ à droite de la route d'accès. Cet emplacement avantageux contribue à la majesté du pavillon, mais permet surtout de pourvoir tant aux besoins du garde La Fontaine qu'à ceux de la suite des chasses lors des haltes : laiterie, cuisines, local pour garder le gibier, etc. y étaient aménagés. Le pavillon du Butard jouit ainsi d'une certaine autonomie de fonctionnement, qui rappelle les dispositions de véritables ermitages tels celui de Madame de Pompadour à Fontainebleau, construit par Ange-Jacques Gabriel en 1749. Témoignage indirect des collations de la suite des chasses du Roi, deux des toiles de la salle à manger des petits appartements de Louis XV à Fontainebleau, Un déjeuner de chasse par JeanFrançois de Troy et une Halte de chasse par Carle Van Loo datés de $1737^{7}$, rendent compte de la dimension sociale de cette activité qui nécessitait des aménagements permettant de recevoir. Le rapprochement s'établit ensuite dans le soin apporté à la décoration intérieure et sa thématique séduisante, qui ne sont peut-être pas étrangers à la présence de la favorite au château voisin de La Celle Saint-Cloud (entre 1748 et septembre 1750). Cependant, si Madame de Pompadour a pu influencer les dispositions du Butard pour lui donner l'apparence d'un lieu de retraite, ce n'est que sous le Consulat que le pavillon est utilisé de façon certaine comme lieu de retraite discret, et parfois à des fins galantes, ainsi qu'en témoigne la comédienne Mademoiselle George ${ }^{8}$. L'ameublement participe également de ce rapprochement entre les usages de pavillon de chasse et d'ermitage. Les premières commandes interviennent en décembre 1751 et janvier 1752, avec la fourniture, par le marchand-mercier Lazare Duvaux, de lanternes, grilles, tables de jeu et chaise d'affaire pour la somme de 2103 livres9. L'ébéniste de la Couronne, Gilles Joubert, est également sollicité entre 1752 et 1761 pour fournir des commodes en bois de noyer qui sont paradoxalement livrées pour le pavillon, tandis qu'il exécute pour le logement du concierge une commode de bois d'amarante ${ }^{10}$. Certains meubles portent la marque "W" du château de Versailles : le Butard étant administrativement rattaché au département des "Dehors de Versailles ", il a pu bénéficier de prêts de faible valeur provenant du château ; seuls les linges fournis portent la marque " $\mathrm{PB}$ » couronnée, et les meubles plus précieux sont inventoriés sans marque aucune. La comparaison des inventaires successifs fait état d'un ameublement relativement immuable au cours des règnes de Louis XV et de Louis XVI, et donc de la permanence d'un ensemble relativement recherché - sans être ostentatoire - dans un pavillon de chasse, avec

6. Ces communs ont subi des transformations au cours du XIXe siècle. Ne subsiste des aménagements primitifs que la deuxième maison du garde à droite en abordant le pavillon. La seconde fut édifiée sous le Second Empire.

7. Ces deux tableaux sont aujourd'hui conservés au musée du Louvre.

8. P.-A. Cheramy, Mémoires inédits de Mademoiselle George, Paris, Plon, 1908 (2édition), pp. 107-112. La rencontre a lieu le 22 octobre 1803.

9. Louis Courajod, Livre-journal de Lazare Duvaux, Paris, Société des bibliophiles français, 1873. 10. Paris, Archives nationales $\mathrm{O}^{1} 3315$ fo $104, \mathrm{O}^{1} 3316$ fo 44 et $\mathrm{O}^{1} 3317$ fo 129. 
où l'étiquette demeure : dans le salon un fauteuil surhaussé est destiné au botté du Roi.

Relativement rapide et soignée, la construction du pavillon du Butard répond aux intérêts de Louis XV pour la chasse et l'architecture. Ange-Jacques Gabriel, en proposant un plan novateur et caractérisé, incite le Roi à construire peu après un pavillon semblable en forêt de Saint-Germain-en-Laye.

\section{Le pavillon de la Muette, copie inaboutie du Butard}

La décision de construire un nouveau rendez-vous de chasse en forêt de SaintGermain intervient après son extension en 1752 au nord de l'étoile ${ }^{11}$ de la Muette.

Fig. 3.

Plan du rez-de-chaussée avec les entresols du pavillon de la Muette dans la forêt de Saint Germain À Versailles ce 19 mars 1774. [Signé] Gabriel Rez-de-chaussée, retombe ôtée Annoté : «Bon à exécuter De la main de M. le D.G. A Versailles le 27 avril $1774 »$ Encre et lavis Paris, Archives national Archives nationales de Pierrefitte-sur-Seine cote $\mathrm{O}^{1} 1722(2)-33$

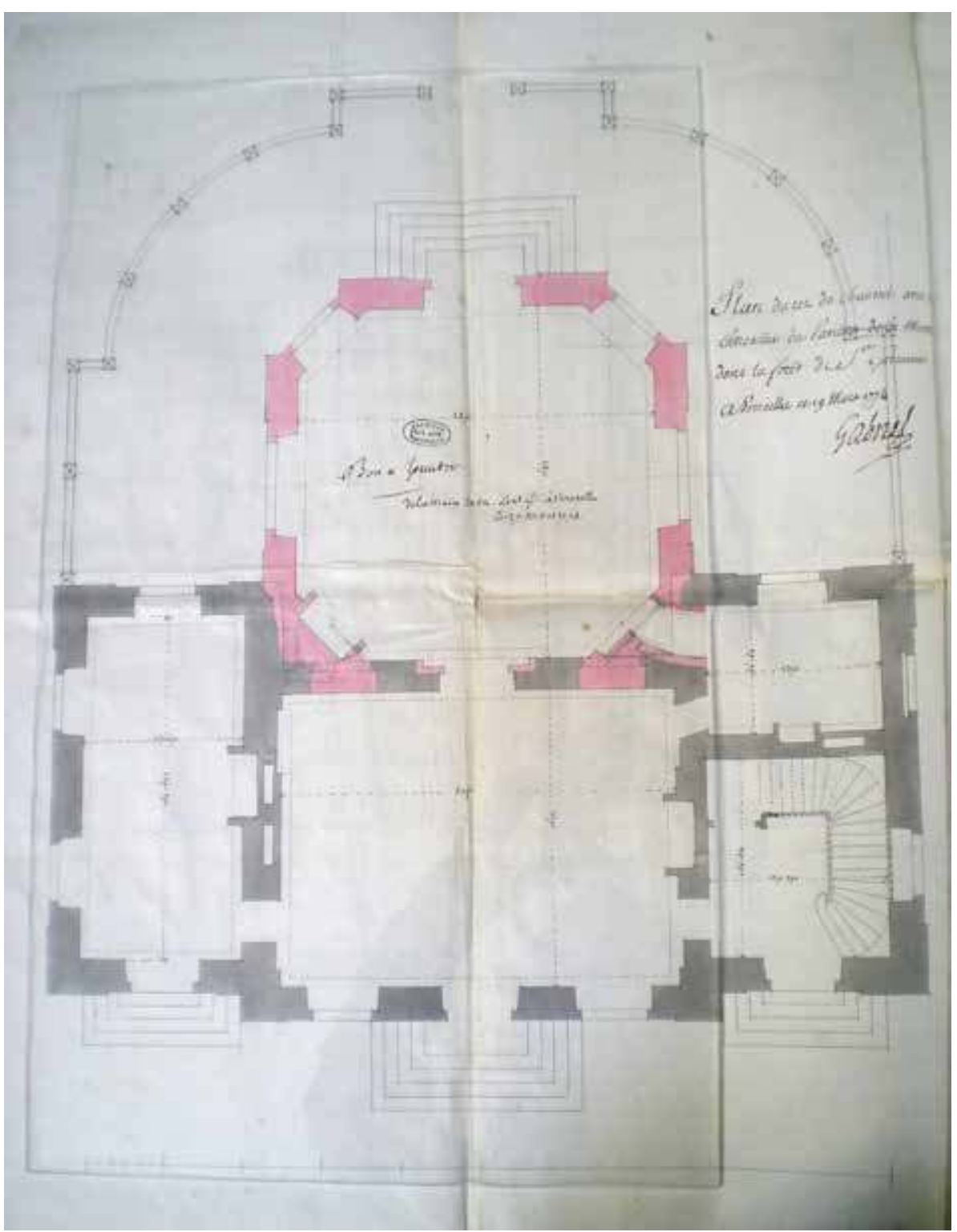

Le premier projet, pensé en 1764 par Ange-Jacques Gabriel, ne verra pas le jour. Il devait être l'héritier, dans sa fonction et dans son plan, du premier château de la Muette construit pour François I $^{\text {er }}$ en 1542 par Pierre Chambiges. En effet, il était prévu d'ériger les murs sur les fondations de l'ancien château, dégagées la même année $^{12}$. Le plan centré du pavillon s'organisait autour d'une salle principale centrale, cantonnée de quatre pavillons carrés en saillie; la perspective sur la

11. On nomme « étoile», un carrefour circulaire où convergent les routes rectilignes ouvertes pour la chasse.

12. L'édifice, déjà très endommagé, fut démoli sur ordre de Louis XIV. Israël Sylvestre en donna peu avant une illustration des plus évocatrices : Paris, Bibliothèque nationale, Estampes, Topo Va 78c, fo I t. I, B8507. 
forêt était déjà importante. Probablement pour des raisons financières, ce parti fut rapidement abandonné au profit de nouvelles dispositions qui, s'appuyant toujours, mais différemment, sur les anciennes fondations, devait être un autre Butard, aux proportions plus vastes.

Les nouveaux plans sont tracés par Gabriel en 1766. Sur les caves aménagées à l'aide des fondations de l'ancien château, la distribution supérieure s'ordonne selon le schéma du Butard, avec quelques légères variantes: le vestibule dans l'avant-corps en légère avancée, donne accès, à droite, cette fois, à l'escalier et à la garde-robe, à gauche à une grande pièce servant aux officiers, et, en face de l'entrée, au grand salon octogonal à l'italienne aménagé dans la forte saillie sur la façade arrière. L'étage abrite trois pièces (fig. 3 et 4). Tandis qu'au Butard la façade est ornée de sculptures, l'extérieur du pavillon de la Muette est beaucoup plus sobre. De son "modèle ", la Muette ne conserve que les chaînes d'angles à bossages, la modénature en bossages en tables continues sur la façade du salon, et les grandes baies cintrées inscrites dans un renfoncement rectangulaire, qui font la signature de Gabriel. Si ces légères variations introduisent une première nuance dans le phénomène de modèle et de copie, une deuxième nuance apparaît avec

Fig. 4.

Pavillon de la Muette Façade d'entrée (sud) et arrière (angle nord-ouest)

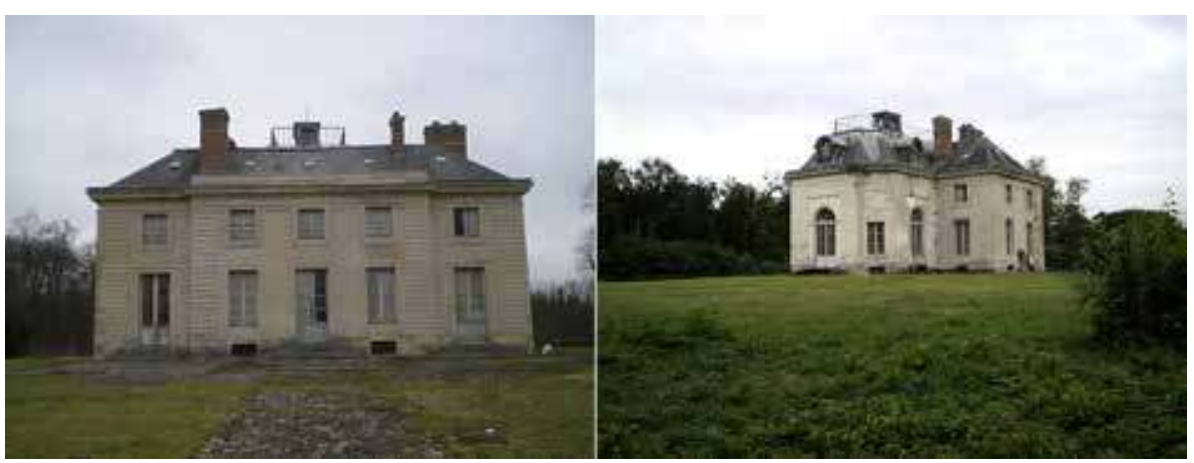

l'aboutissement à la Muette de projets esquissés sur certains plans du Butard : l'absence de fronton, le toit brisé en pavillon couvrant le grand salon, ou encore la communication entre ce dernier et le cabinet de chaise montre l'interdépendance des plans tracés par Gabriel. Un facteur géographique accentue la singularité du pavillon de la Muette : à l'inverse du Butard, soumis au tissu boisé alentour, la Muette, érigé au centre d'un carrefour, dirige l'organisation de la forêt proche. À partir du centre du salon, et par l'intermédiaire de ses baies, de nouvelles routes rayonnantes sont percées, ainsi que l'annonce le contrôleur des Bâtiments au département de Saint-Germain-en-Laye dans une lettre adressée Marigny ${ }^{13}$, directeur des Bâtiments :

«Messieurs de la Maîtrise des Eaux et Forêts de Saint-Germain m’ont lu un arrêt du Conseil [...] en joignant un emplacement pour le logement d'un concierge, et des routes neuves à percer, dont le point central partira du milieu du salon de Sa Majesté ; comme il faudra que les rayons percés du milieu des croisées prolongent les routes dans leurs étendues, savoir de Monsieur le marquis si je puis me prêter à l'exécution de cette besogne ${ }^{14}$ ».

Ce caractère panoramique est renforcé par la construction d'un belvédère sur la toiture. Étudiant le comportement de Louis XV au cours d'une de ses visites de chantier, le contrôleur de Saint-Germain eut l'idée de ce dispositif d'observation utile pour organiser la chasse, et qui rappelle qu'à Versailles, le Roi dispose d'agréments semblables. Depuis ce belvédère, la vue sur la forêt et les routes rayonnantes est aujourd'hui encore impressionnante. Ainsi mis en valeur

13. Abel-François Poisson de Vandières, marquis de Marigny, directeur des Bâtiments du Roi de 1751 à 1773 .

14. Paris, Archives nationales, $\mathrm{O}^{1} 1712$ - 580, lettre du 7 novembre 1767 . Deux plans montrent les travaux. Le premier est daté du 22 février 1767. L'autre, daté du 11 juillet 1767, définit le terrassement à effectuer : «Plan coté, et projet pour agrandir le carrefour de la Meutte [sic], élargir la principale route qui traverse ledit carrefour, former un emplacement pour établir un logement d'un Inspecteur des chasses, ériger de nouveaux carrefours, demi-lune, percer des nouvelles routes, et en redresser une ancienne, le tout pour faciliter les abords du nouveau pavillon pour les rendez-vous des chasses du Roi » (Paris, Archives nationales, $\mathrm{O}^{1} 1722$ (2) - 12 et $\left.\mathrm{O}^{1} 1722(2)-11\right)$. 
au centre d'un grand carrefour circulaire, le pavillon est également visible depuis les routes convergentes, et notamment depuis la grande route d'accès venant de Saint-Germain-en-Laye. Enfin, le facteur économique a lourdement pesé sur l'aspect du pavillon de la Muette. L'importance du site devait être renforcée par la construction à droite d'un grand ensemble de communs abritant logement du garde et écuries, comme au Butard ; faute de moyens, il ne fut jamais réalisé sous l'Ancien Régime, mais les plan $s^{15}$ ne laissent pas de montrer ici un nouveau parallèle entre les deux pavillons.

Le chantier de la Muette est confié au contrôleur de Saint-Germain-en-Laye, Nicolas Galant, homme zélé qui a laissé une correspondance abondante ${ }^{16}$, parfois piquante, qui relate par le menu l'avancement des travaux. Contrairement à celui du Butard, le chantier du pavillon de la Muette a été très lent, retardé notamment par les réticences des ouvriers : "qui reculent à venir à un atelier isolé et éloigné des secours de la vie et du couvert pour se $\log e^{17}{ }^{17}$ ». De fait, l'exécution du grand salon octogonal en saillie sur la façade arrière est rapidement suspendue, et ne sera entreprise que peu de temps avant le décès de Louis XV. Seul Louis XVI verra le pavillon dans la configuration globale souhaitée par son grand-père.

La plus grande vigilance quant aux coûts, et le possible désintérêt de Louis XVI pour ce pavillon (qu'il occupe régulièrement néanmoins ${ }^{18}$ ) achèvent de faire de la Muette une copie inaboutie et délaissée du Butard. Le comte d'Angiviller ${ }^{19}$, alors directeur des Bâtiments, modère l'enthousiasme de Galant, et, tout en le poussant à achever promptement le gros œuvre, l'engage à veiller à la dépense : "dans un lieu de cette nature où tout ornement est superflu ${ }^{20}$ ", il semble qu'il n'y ait jamais eu de glaces sur les cheminées, dont la plupart sont de remploi ${ }^{21}$. Toutes les boiseries du pavillon, sobrement sculptées à grands cadres par Guesnon et Clicot en 1768 (auteurs des boiseries du Petit Trianon l'année précédente), ne sont posées qu'à l'achèvement du grand salon, en 1775 . Enfin, en 1778, il est décidé de faire du premier étage un logement pour le garde Brou, puisque le pavillon ne dispose d'aucune dépendance hormis quelques appentis pour le bétail.

L'ameublement est extrêmement simple et rare, contrastant avec l'intérêt que portait Louis XV à son nouveau rendez-vous; seules les armes du Roi sur les chenets du salon et les deux chaises de paille utilisées pour son botté indiquent sa destination royale. À la même époque rappelons-le, le Butard était meublé avec plus de recherche, par les soins, notamment, de Lazare Duvaux. Il faut attendre l'achèvement du grand salon sous le règne de Louis XVI pour trouver une modeste augmentation de mobilier. Jean-Henri Riesener est chargé de la fourniture d'une table de piquet en acajou en $1783^{22}$, mais, dans l'ensemble, l'ameublement ne présente rien d'aussi remarquable qu'au pavillon du Butard.

Le pavillon de la Muette de Louis XVI « devant présenter plus de commodités que de magnificence ${ }^{23}$ " ainsi que le préconisait d'Angiviller, est donc, par une somme de facteurs relevant tant des finances, de l'intérêt royal, du décès de Louis XV son initiateur, que des vues parfois opposées du directeur et du contrôleur, une copie améliorée mais inaboutie du Butard. Le Roi et son architecte ont-ils pour autant voulu " copier " littéralement ce dernier? En l'absence de témoignage écrit, il est sans doute plus juste de supposer que, fort des dispositions

15. Paris, Archives nationales, $\mathrm{O}^{1} 1722$ (2) - 11, - 16, - 17, - 18, - 29, - 31, - 32, - 36. 16. Cette correspondance est conservée aux Archives nationales sous les cotes $\mathrm{O}^{1} 1712$ à $\mathrm{O}^{1} 1715, \mathrm{O}^{1} 1717$. Quelques documents ont été joints aux archives concernant le château de la Muette du bois de Boulogne, rassemblés sous la cote $\mathrm{O}^{1} 1582$.

17. Paris, Archives nationales, $\mathrm{O}^{1} 1712-467$, lettre de Galant à Marigny en date du 14 septembre 1766.

18. Ainsi qu'en attestent la correspondance et le Journal de Louis XVI, Paris, Archives nationales, AE I 4.

19. Charles-Claude Flahaut de La Billarderie, comte d'Angiviller, directeur des Bâtiments du Roi de 1774 à 1789 .

20. Paris, Archives nationales $\mathrm{O}^{1} 1717$ (5), réponse de d'Angiviller à Galant en date du 13 novembre 1775 .

21. Ainsi qu'en atteste I'« État des vieux chambranles de cheminées de marbres de démolitions qui sont dans les magasins du Roi, et choisis pour le revêtement des cheminées du pavillon de la forêt qui va servir de rendez-vous de chasse pour Sa Majesté à Saint-Germain », Paris, Archives nationales, $\mathrm{AN} \mathrm{O}^{1} 1717$ (4), lettre de Galant à Marigny en date du 22 août 1767).

22. Paris, Archives nationales, $\mathrm{O}^{1} * 3628$.

23. Paris, Archives nationales, $\mathrm{O}^{1} 1717$ (5), lettre de Galant à d'Angiviller en date du 5 novembre 1775. 
agréables du Butard, Gabriel a voulu donner au Roi un second pavillon qui s'en inspirait, ce que montre l'exécution à la Muette des projets non réalisés au premier, et l'adaptation de son architecture au tissu forestier quadrillé de routes rectilignes.

\section{Le devenir des pavillons au XIX ${ }^{e}$ siècle}

Après leur construction, les deux pavillons ne connaissent plus de travaux d'envergure ; ainsi, leur configuration du XVIII ${ }^{\mathrm{e}}$ siècle subsiste encore aujourd'hui. Après la Révolution, et leur reconversion en résidence particulière, ils renouent avec leur destination initiale: Napoléon Bonaparte et les chefs d'état suivants les utilisent comme rendez-vous de chasse. Preuve de leur utilité et de leur fréquentation, les architectes sont sollicités sur la question des communs, et le Garde-Meuble sur celle de l'ameublement.

En effet, si la question de l'architecture des pavillons ne relève plus, au $\mathrm{XIX}^{\mathrm{e}}$ siècle, que de l'entretien voire de quelques améliorations topographiques, l'augmentation progressive et la relative richesse de l'ameublement dénotent une volonté de faire de ces deux rendez-vous de chasse des haltes d'envergure propres à recevoir. Cet ameublement n'est jamais homogène, et fait se côtoyer tant le remploi que les créations contemporaines de l'époque et spécifiques à ces bâtiments. L'Empire privilégie la création artistique afin de relancer l'économie du pays, mais il puise aussi dans le fonds d'Ancien Régime, conservé au GardeMeuble, pour faire face aux nécessités d'ameublement croissantes après les troubles de la Révolution. C'est ainsi que le " feu au dromadaire " provenant du cabinet turc de Marie-Antoinette à Fontainebleau ${ }^{24}$, est installé dans le grand salon du pavillon de la Muette de 1808 à $1830^{25}$. D'après les descriptions fournies dans l'inventaire, et une gravure de journal ${ }^{26}$, le pavillon a été meublé sous la monarchie de Juillet en partie de remplois Empire, et en partie de créations contemporaines fournies notamment par Alphonse Jacob Desmalter, Louis-Édouard Lemarchand et Pierre-Philippe Thomire. Aucun de ces meubles n'a pu à ce jour être identifié : après leur retour au Garde-Meuble en 1852, toute trace d'eux disparaît des registres, et l'on ignore, en l'état actuel des connaissances, s'il existait une marque spécifique à ce pavillon.

Comme au pavillon de la Muette, un ameublement en partie Empire est réutilisé au Butard sous la monarchie de Juillet. Il subsiste sous le Second Empire, et cette permanence a joué en faveur de l'identification de certaines pièces dans les collections publiques françaises. C'est ainsi que furent installés des sièges de François-Honoré-Georges Jacob Desmalter, Denis-François Frémancourt, Pierre-Benoît Marcion, Jean-Pierre Louis, ou encore des flambeaux de LouisIsidore Choiselat ${ }^{27}$. L'identification de ces sièges et objets revêt une importance particulière : ils portent une marque spécifique au pavillon, un "VB » inscrit à l'encre, à comprendre comme "Versailles Butard». La datation de cette marque reste incertaine, mais on peut la situer après 1838 (une commode signée Alexandre-François Maigret, ayant quitté le pavillon cette année-là, ne la porte $\mathrm{pas}^{28}$ ), ou plus probablement sous le Second Empire. Malgré cette incertitude, elle indique le rattachement du Butard au domaine de Versailles, tout comme il l'était sous l'Ancien Régime, englobé dans le département des "Dehors de Versailles" (fig. 5).

24. Musée du Louvre, inv. OA 5260.

25. Inventaires du pavillon de la Muette de 1808, (Paris, Archives nationales $O^{2}$ 695, et de 1818 , id. $\mathrm{AJ}^{19} 451$. Il entre au Garde Meuble le 29 novembre 1830 (id. AJ ${ }^{19}$ 622).

26. Paris, Archives nationales $A J^{19} 450 / 2$, et Paris, Bibliothèque nationale de France, Va 78 c. fol tome 1 B8514 (cette gravure est probablement tirée de L'Illustration).

27. L'imposant ensemble de sièges de Jacob Desmalter compte deux canapés, deux causeuses et huit fauteuils, aujourd'hui déposés par le musée national des châteaux de Versailles et de Trianon au Sénat. Ont aussi été identifiés : cinq chaises de Frémancourt, une bergère et cinq fauteuils de Marcion, et deux chaises de Louis (Paris, Mobilier national). La paire de flambeaux de Choiselat est conservée au musée des châteaux de Versailles et de Trianon.

28. Conservée au musée des châteaux de Versailles et de Trianon. 

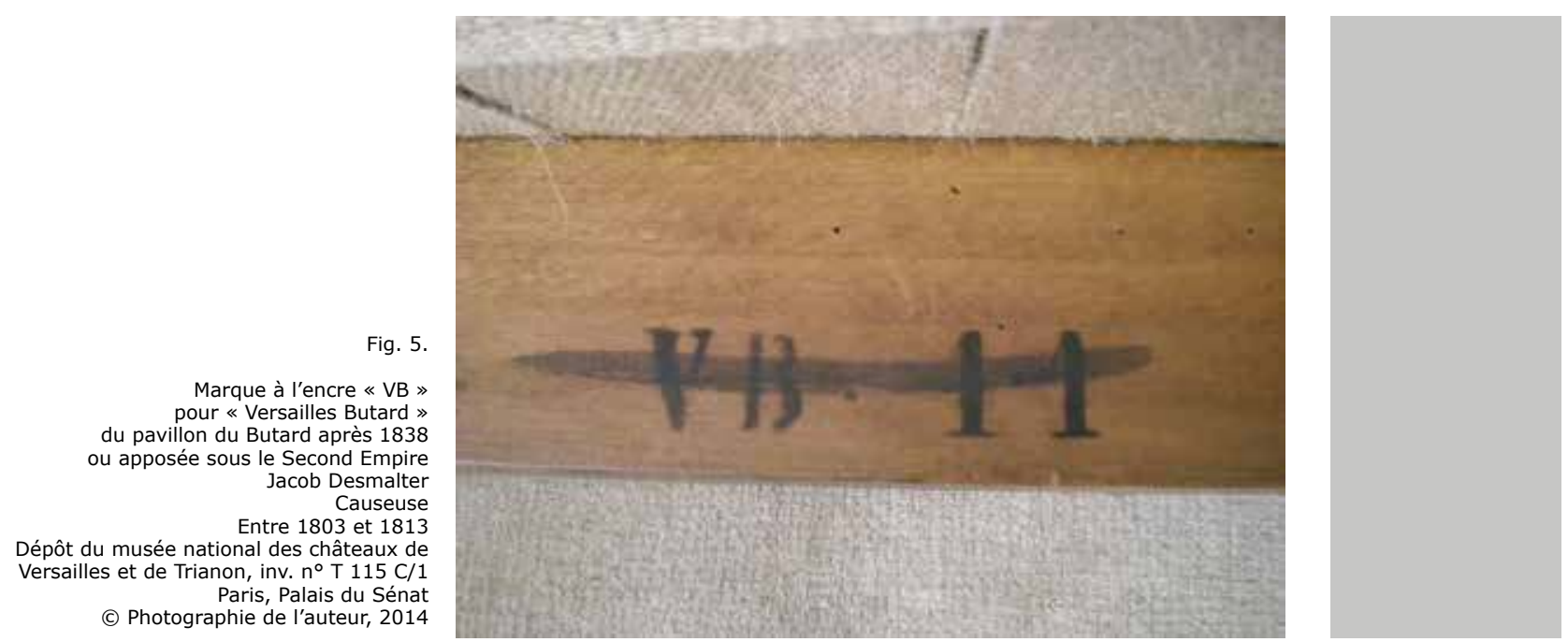

Si la monarchie de Juillet est la période où les identifications de meubles sont les plus nombreuses, il revient au Consulat d'apporter des témoignages de commandes spécifiques au pavillon du Butard, bien que les inventaires dressés sous ce régime ne soient pas conservés. Une chaise et un fauteuil signés Jacob frères, rue Meslée $e^{29}$ n'auraient pu être situés au pavillon du Butard si deux étiquettes manuscrites n'avaient été conservées sur les traverses ${ }^{30}$ (fig. 6). Le rachat du Butard et de ses bois environnants par Bonaparte en 1802 motive une campagne de remeublement, ainsi qu'il l'ordonne dans toutes les demeures officielles; ces deux sièges, particulièrement caractéristiques du Consulat et du style dit « retour d'Égypte " pour le fauteuil, laissent à penser que le pavillon fut à cette époque meublé à la dernière mode, bref moment d'homogénéité qui ne se retrouvera plus par la suite.

Fig. 6.

Jacob frères. Rue Meslée Étiquette de provenance originelle Chaise
$1802(?)$
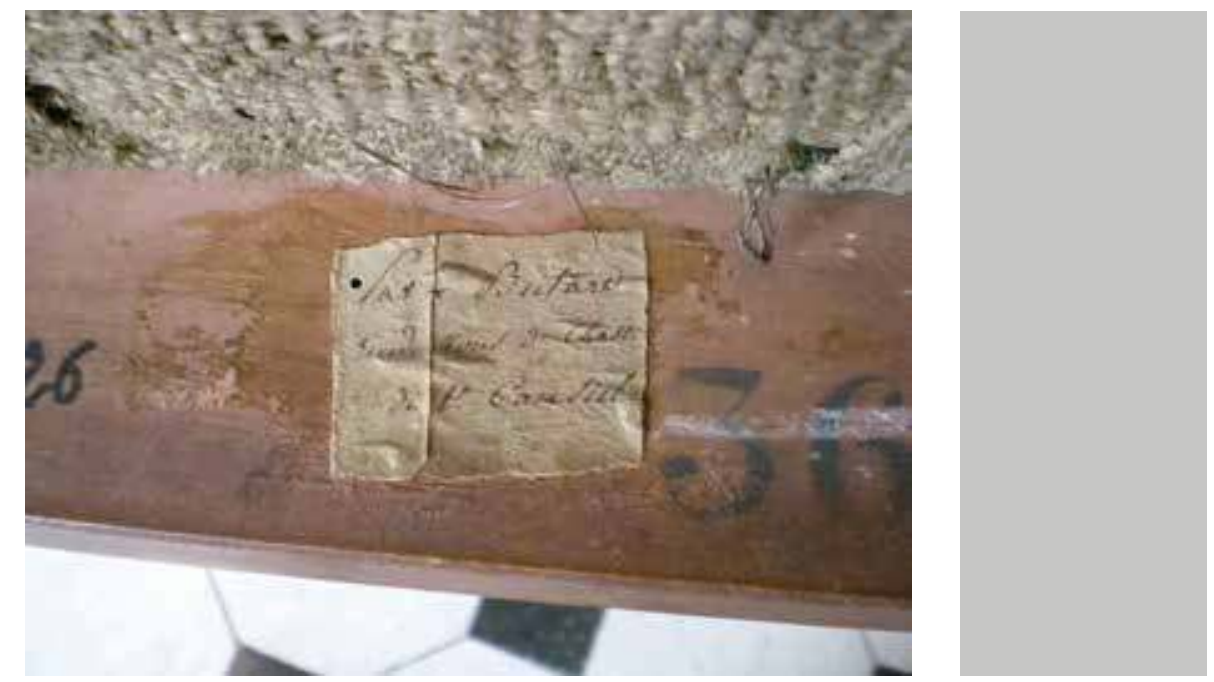

Des liens architecturaux et topographiques indéniables lient les pavillons du Butard et de la Muette depuis leur construction. Ange-Jacques Gabriel, qui annonce avec le premier à la fois son style en devenir et le néoclassicisme, fournit pour les nécessités de la chasse une disposition caractérisée, dont le plan en T avec salon saillant sur la façade arrière est le trait le plus remarquable. La relation de modèle et de copie qu'ils entretiennent est à imputer à la commodité d'un tel plan : le plan rectangulaire du pavillon de Verrières (1750), autre rendez-vous de

29. Ils sont respectivement conservés au musée national des châteaux de Malmaison et de BoisPréau et au Mobilier national.

30. La chaise est signalée par Dr Ed. Christen, « Notes d'histoire : Le Butard », Bulletin de la Société archéologique Historique et Artistique de Marly-le-Roi, 1932, t. I, n² 2, p. 6, puis M. Noury, «Recherches sur Le Butard », Bulletin de la Société archéologique Historique et Artistique de Marly-le-Roi, 1939, t. I, n 8, pp. 237-240. Le fauteuil a été signalé à I'auteur par Jean-Jacques Gautier, inspecteur au Mobilier national. 
Gabriel aujourd'hui disparu, "aussi beau, aussi grand et aussi commode que celui du Butard ${ }^{31}$ » n'a pourtant pas été retenu pour l'édification de la Muette, ni même le premier projet de 1764. Cette relation de copie est cependant à nuancer. Si le soin apporté à l'élaboration du Butard, et cette double fonction utilitaire et sociale, l'associent à un petit ermitage, les spécificités des lieux, les aléas financiers et les divergences d'opinions introduisent des différences notables et durables entre les deux pavillons. Le pavillon de la Muette, en subissant les décisions restrictives de l'administration, dans le même temps qu'il profite d'agréments uniques comme le belvédère, apparaît ainsi plus comme un rendez-vous conçu pour la chasse exclusivement que comme un ermitage. Il est donc à ce titre plus juste de parler d'interprétation et d'adaptation. Contrairement à d'autres bâtiments, le Butard et la Muette échappent tous deux aux destructions révolutionnaires, pour retrouver leur destination initiale, et, par leurs ameublements croissants en quantité et en qualité, un statut de rendez-vous de chasse qu'ils garderont jusqu'à la chute du Second Empire, tant l'activité cynégétique est liée au pouvoir.

\section{L'auteur}

Ancienne élève de l'École du Louvre (premier et deuxième cycles), Marie-Marguerite Roy s'est spécialisée dans l'étude de l'architecture et des arts décoratifs français du $\mathrm{XVIII}^{\mathrm{e}}$ et de la première moitié $\mathrm{du} \mathrm{XIX}^{\mathrm{e}}$ siècle. Après son mémoire de Master 1, portant sur les appartements de Louis XV et Marie Leszczynskà à Compiègne, elle a choisi de se consacrer en 2012 aux pavillons de chasse de Louis XV et Louis XVI, dont deux ont fait l'objet de son Master 2. 